\title{
Computational Design, Synthesis and Photochemistry of Cy7-PPG, an Efficient NIR-activated Photolabile Protecting Group for Therapeutic Applications
}

\author{
Georgios Alachouzos, ${ }^{\star}$ Albert M. Schulte, Anirban Mondal, Wiktor Szymanski, ${ }^{*}$ Ben L. \\ Feringa* \\ AUTHOR ADDRESS - Centre for Systems Chemistry, Stratingh Institute for Chemistry, Faculty \\ for Science and Engineering, University of Groningen, Nijenborgh 4, 9747 AG Groningen, The \\ Netherlands; and Department of Radiology, Medical Imaging Center, University Medical \\ Center Groningen, University of Groningen, Hanzeplein 1, 9713 GZ Groningen; The \\ Netherlands
}

KEYWORDS: Photopharmacology, Photolabile Protecting Groups, Photochemistry, DFT,
NIR light

\begin{abstract}
Photolabile Protecting Groups (PPGs) are chemical tools used in photopharmacology for the oxygenindependent release of bioactive payloads, enabling spatiotemporal activation of their bioactivity. However, red-shifting of PPG activation wavelengths into the NIR range, light which penetrates the deepest in tissue, has been laborious, and often results in inefficient or insoluble PPGs. This challenge prohibits the use of current photocages in complex biological targets, hindering the progression of photopharmacology into the clinic. To directly address this problem, we report herein a novel concept in PPG design, by transforming clinically-applied NIR-dyes with suitable molecular orbital configurations into new NIR-PPGs using computational approaches. Using this method, we demonstrate how Cy7, a class of clinically-employed NIR dyes possessing ideal properties, such as NIR-absorption, very high molecular absorptivity, and excellent solubility in aqueous media, can be successfully converted into Cy7-PPG. We report a facile organic synthesis towards Cy7-PPG from easily accessible precursors and confirm its function as an oxygen-independent NIR-PPG. Cy7-PPG is the most redshifted PPG to date $\left(\lambda_{\max }=746 \mathrm{~nm}\right)$, with unmatched molecular absorptivity and solubility, and excellent uncaging efficiency, paving the way for the use of PPGs in the clinic.
\end{abstract}

\section{Introduction}

Photopharmacology is the scientific discipline of employing light to control therapeutic action inside a biological specimen or a living system. ${ }^{[1-7]}$ Light is non-invasive to the system, ${ }^{[6-8]}$ and confers unparalleled spatiotemporal control of function, with resolutions in the order of micrometers and milliseconds. ${ }^{[9]}$

Photolabile Protecting Groups (PPGs) are cornerstone molecular tools within photopharmacology (Figure 1a). ${ }^{[10-17]}$ PPGs are small molecules that are covalently bound to a bioactive payload (PL) (a small molecule drug, biological, etc.) to block ("cage") its therapeutic action. Irradiating the PPG at its main absorption band induces the release of the PL, restoring ("uncaging") its bioactivity.

Unsurprisingly, the extent to which PPGs can be deployed in the human body, and therefore be clinically applied, hinges heavily on the photochemical properties of the PPG (Figure 1b). ${ }^{[1,11,13]}$ First, the PPG must absorb light wavelengths that are both benign to human tissue and are deep-reaching. For this purpose, the near-IR (NIR) wavelength range has become synonymous with the "therapeutic window", with 700-800 nm light penetrating the deepest in human tissue. ${ }^{[18]}$ Beyond its absorbance, the ideal PPG must uncage efficiently (i.e. the product of its molar extinction coefficient and its uncaging quantum yield, $\varepsilon \times \Phi_{\text {het }}>100 \mathrm{M} \mathrm{M}^{-1} \mathrm{~cm}^{-1}$ ), ${ }^{[12]}$ and be both soluble and non-toxic to the tissue (Figure 1c). For uncaging to occur without phototoxicity and to allow for PL release in a wide range of biological targets, including hypoxic tissues such as solid tumors, or even bacterial biofilms, the PL release mechanism should be independent of the presence of oxygen. ${ }^{[19,20]}$ 
Figure 1. PPGs in photocaging, current PPG designs and the undiscovered ideal PPG for use in tissue

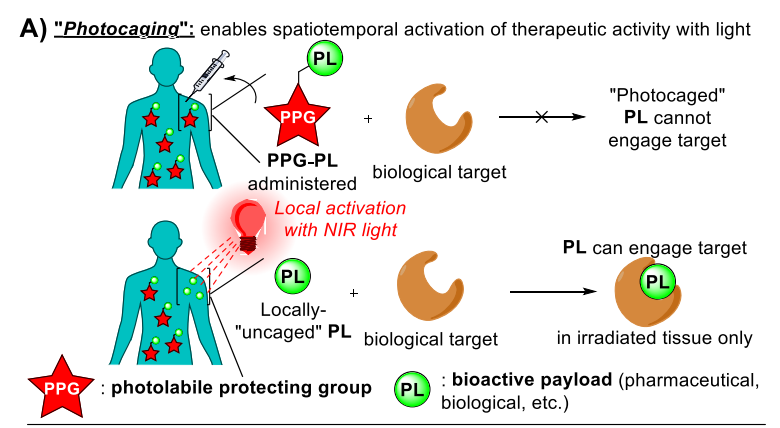

B) The challenge: red-shifting PPGs into the NIR = poor PPG efficiency and solubility

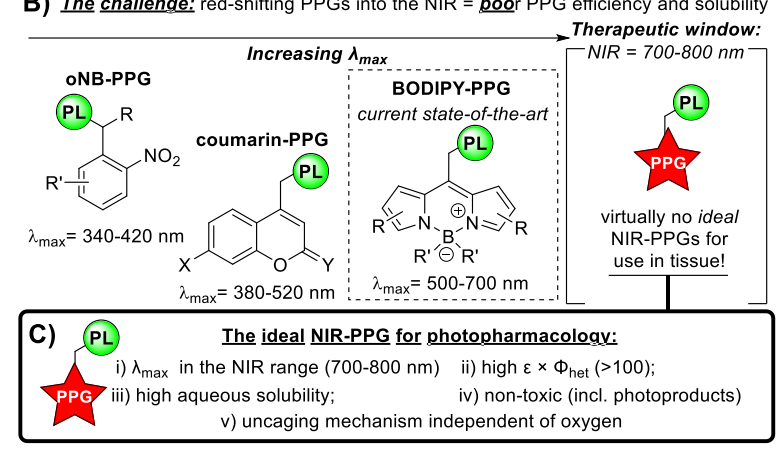

Such an ideal PPG has not yet been discovered, and thus the advancement of PPG therapies into the clinic is currently hampered. ${ }^{[11,12]}$

Though the ideal NIR-PPG remains elusive, tremendous recent advances have been made to "red-shiff" PPGs activation wavelengths from the UV or visible light range towards the NIR-range (Figure 1b). For example, BODIPY-PPG absorption bands have been red-shifted by nearly $200 \mathrm{~nm}$ to generate variants now constituting the "state-of-the-art" in PPGs approaching the therapeutic window. ${ }^{[12,21]}$

However, these red-shifting strategies employed are not without their downsides. Red-shifting PPGs typically involves extending the conjugation of their chromophore, which results in: i) loss of PPG solubility in water due to increased lipophilicity; and ii) decreased uncaging quantum yield ( $\Phi_{\text {het }}$. ${ }^{[10-12,22-}$ ${ }^{24]}$ Beyond being an inherently uphill battle, red-shifting strategies suffer from a multi-parameter "hydra paradox" since improvement towards one ideal property often leads to diminished progress towards another ideal property. ${ }^{[12]}$

As a discipline exiting its infancy and looking to finally emerge in the clinic, ${ }^{[1,2]}$ photopharmacology urgently needs PPGs with well-balanced ideal properties: i) a $\lambda_{\max }$ centered between 700-800 nm; ii) a high uncaging cross-section $\varepsilon \times \Phi_{\text {het; }}$ iii) high solubility in water; iv) low (photo)toxicity; v) oxygenindependent uncaging. Here we describe our computation-driven development of the first NIR-PPG to fully meet these requirements. 


\section{Computational Design}

At the outset of our quest to design a NIR-PPG, we analyzed the fundamental photochemical pathways of existing PPGs. PL uncaging occurs from either the PPG excited singlet $\mathrm{S}_{1}$ state, or its triplet $\mathrm{T}_{1}$ state, ${ }^{[25]}$ assuming that the spin-orbit coupled intersystem crossing (ISC) to this $T_{1}$ state is sufficiently fast. ${ }^{[10,12,23]}$ The desired heterolytic pathways are shown in blue in Figure 2a, where an ion pair of the PL anion and the PPG cation are formed upon heterolysis. Beyond heterolysis, various competing pathways such as radiative (e.g. fluorescence from $S_{1}$ or phosphorescence from $T_{1}$ ) or non-radiative processes (e.g. the phototoxic sensitization of triplet oxygen to singlet oxygen from $T_{1}$, or ion pair recombination) are analogously shown in red. Strategies toward efficient NIR-PPGs should therefore strive to minimize the sum of all these unproductive photochemical processes. ${ }^{[12]}$ After PL heterolysis, successful interception of this cation commonly takes place by the solvent. For example, nucleophilic attack by water results in an alcohol product, which unfortunately often absorbs in the same spectral range as the PPG, competing for the photons. ${ }^{[10-12,22]}$ Novel NIR-PPGs should also disinherit this limitation.[26]

Figure 2. An overview of the function of PPGs and our novel DFT-based workflow for designing ideal NIR-PPGs

a) PPG photochemical pathwavs

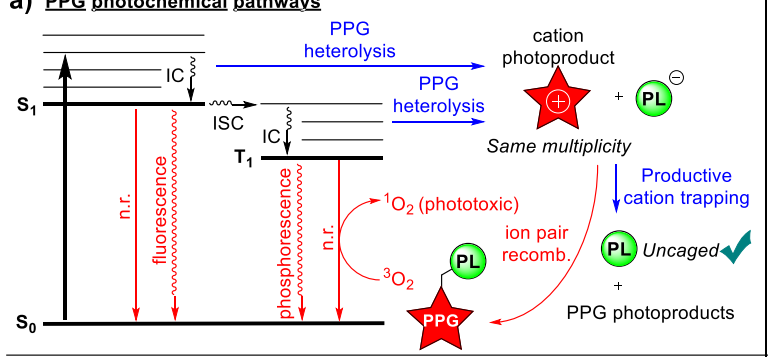

b) $\underline{P P G}$ function from FMO Configurations

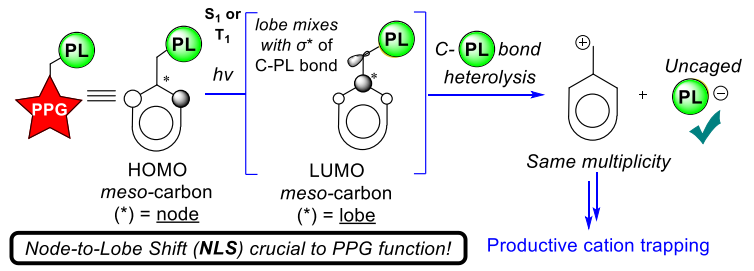

c) A DFT-based computational method for converting NIR-dves into NIR-PPG

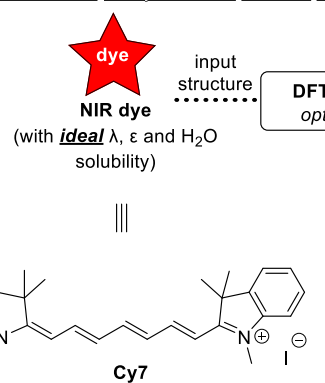

Cy7

$\varepsilon=1.1$ to $2.7 \times 10^{5} \mathrm{M}^{-1} \mathrm{~cm}^{-1}$

$\mathrm{H}_{2} \mathrm{O}$-soluble, non-toxic, biocompatible ${ }^{[7]}$

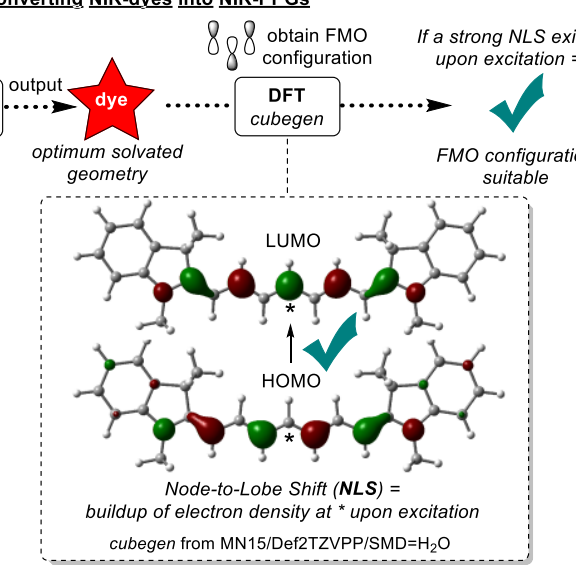

cubegen from MN15/Def2TZVPP/SMD $=\mathrm{H}_{2} \mathrm{O}$

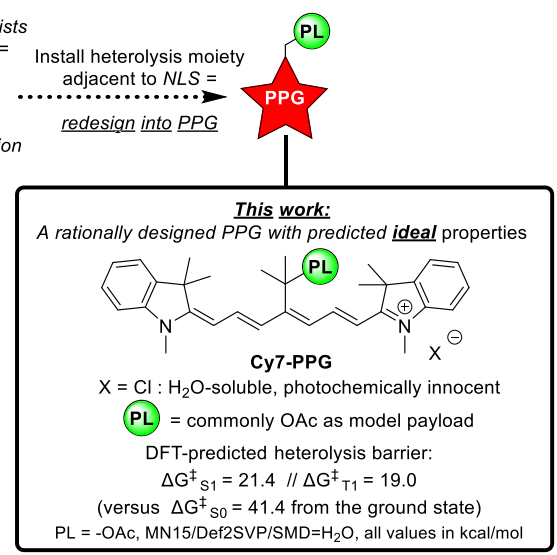

For our design of new NIR-PPGs, we also considered that the heterolytic function of a PPG hinges on the changes of the frontier molecular orbital (FMO) configurations during excitation by light. ${ }^{[10,12]}$ In the ground state, the populated HOMO of a PPG must possess no orbital coefficient (an orbital node) at the meso-carbon (indicated by an * in Figure $2 b$ ), thus positioning no electron density $\beta$ to the leaving group PL. The ground state FMO configuration thus prevents heterolysis in the absence of irradiation. Conversely, excitation of the PPG chromophore to either the $S_{1}$ (or $T_{1}$ state after ISC) populates the LUMO configuration, which now possesses a high orbital coefficient (an orbital lobe) at the meso-carbon. This excited state FMO configuration thus positions a high electron density $\beta$ to the leaving group PL. Consequently, this newly populated LUMO mixes with the $\sigma^{*}$-orbital of the C-PL $\sigma$-bond, which results in the breaking of the bond and ultimately drives heterolysis of PL.

We have considered this FMO Node-to-Lobe Shift (NLS) as a crucial functional element for our forward engineering of the ideal NIR-PPG. We hypothesized that existing NIR-dye scaffolds that absorb a sufficient amount of light at an appropriate wavelength (namely, 700-800 nm) and have a well-defined NLS in their FMO configurations could be engineered into PPGs by inclusion of a key functional moiety: 
a PL leaving group $\beta$ to their NLS. Since the introduction of this moiety is a minimal molecular modification, one would expect the properties $\left(\lambda_{\max }, \varepsilon\right.$, aqueous solubility, biocompatibility etc.) of the starting NIR-dye to be largely conserved in the engineered NIR-PPG. This design principle liberates us from starting our search for ideal PPG among the typical PPG scaffolds (Figure 1) and instead allows us to engineer PPG functionality into NIR-dyes already possessing ideal properties. To the best of our knowledge there are no reported cases of the NLS being deliberately utilized towards the development of NIR-PPGs from NIR-dyes, suggesting that this FMO approach towards NIR-PPGs is unexploited.

This design approach culminated in a computational workflow (Figure 2) for obtaining the FMO configurations from the DFT-optimized structures of potential NIR dye candidates with ideal properties $\left(\lambda_{\max }, \varepsilon\right.$, aqueous solubility, biocompatibility etc.). With this method, the candidate dyes' FMO configurations are extracted from the DFT wavefunctions obtained at the MN15 / Def2TZVPP / $\mathrm{SMD}=\mathrm{H}_{2} \mathrm{O}$ level (see SI for further computational details). The combination of global-hybrid exchangecorrelation functional with the minimally-augmented triple- $\zeta$ basis set and the Solvation Model based on Density (SMD) method was expected to give a good balance of predictive accuracy versus computational cost. ${ }^{[27-29]}$ Thereafter, the dye FMO configurations can be conveniently screened for the existence of an NLS. Indeed, we demonstrate here that if a strong NLS is present in a NIR-dye FMO configuration, the dye can be redesigned into a PPG.

One such NIR-dye that was examined was Cy7, a blockbuster fluorescent heptamethine dye used extensively in biology (Figure 2c). ${ }^{[30,31]}$ Cy7 has a $\lambda_{\max }$ of $736 \mathrm{~nm}$, precisely in the therapeutic window, and also exhibits a molecular absorptivity $\varepsilon$ of the order of $10^{5} \mathrm{M}^{-1} \mathrm{~cm}^{-1}$ in aqueous media. ${ }^{[32-34]}$ Furthermore, Cy7 and the broader family of heptamethine dyes have extensive clinical applications, such as NIR-imaging and photodynamic therapy.[30,35] As a result, heptamethine dyes and their photoproducts have been well studied in terms of their in vivo effects, and are generally regarded to be safe for use in humans. ${ }^{[30]}$ In sum, Cy7 is a privileged starting point for the design of ideal PPGs for use in the clinic.

In fact, heptamethine chromophores have been successfully incorporated into photorelease systems that do not proceed via simple heterolytic step, as the PPGs previously discussed (Figure 2b). ${ }^{[36,37] \text { In }}$ these seminal systems, the photorelease of PLs was achieved by harnessing the oxygen photosensitizer properties of heptamethine dyes ( $\left.\Phi_{\text {P.S. }}<0.5 \%\right)$ excited by $690 \mathrm{~nm}$ light. Unfortunately, a downside is that it requires singlet oxygen for its release mechanism, and thus PL release is not uncoupled from phototoxicity. This also prohibits the applications of these photocaged systems in hypoxic tissues. ${ }^{[19,20]}$ Lastly, the $\lambda_{\max }$ of this photorelease system is centered outside the ideal wavelength range of $700-800 \mathrm{~nm} .{ }^{[18]}$ Thus, the ideal PPG based on heptamethine dyes such as Cy7 is yet undiscovered.

Employing the computational workflow shown in Figure 2, Cy7 was indeed found to have a strong NLS at its meso-carbon. In our redesign of Cy7 into a PPG, we first elected to introduce a 2-substituted propanol subunit as the heterolytic moiety, positioning a $\beta$-leaving group adjacent to the NLS. Next, we considered the heptamethine cation counterion. Cy7 as the iodide salt already enjoys superior solubility in water compared to many fluorescent dyes. ${ }^{[34]}$ Nonetheless, we instead sought to develop this PPG as the chloride salt, as chloride is photochemically innocent and should also further increase the PPG's water solubility versus iodide. ${ }^{[38-40]}$ These combined design elements allowed us to redesign NIR-dye Cy7 into Cy7-PPG (Figure 2).

An additional support for our PPG design came from the computed thermochemistry of heterolysis from the TD-DFT excited states of Cy7-PPG. At the TD-MN15/Def2SVP/ SMD= $\mathrm{H}_{2} \mathrm{O}$ level, with $\mathrm{PL}=\mathrm{AcOH}$ (Figure 2c) as the archetypal model PPG leaving group, ${ }^{[21,41]}$ a modest barrier for heterolysis from the excited $S_{1}$ state $\left(\Delta \mathrm{G}^{\mathrm{S}_{1}}=21.4 \mathrm{kcal} / \mathrm{mol}\right)$ was found. Furthermore, since the parent dye Cy7 undergoes rapid ISC to its low lying triplet $T_{1}$ state, ${ }^{[32-34]}$ the thermochemistry of heterolysis of Cy7-PPG from $T_{1}$ was computed as well. We were pleased to find an even lower barrier of $\Delta G \ddagger_{T 1}=19.0 \mathrm{kcal} / \mathrm{mol}$ for the $\mathrm{T}_{1}$ heterolysis. Gratifyingly, the analogous computed heterolysis barrier from the ground state $\mathrm{S}_{0}$ was found to be prohibitively high $\left(\Delta \mathrm{G}^{\ddagger} \mathrm{s} 0=41.4 \mathrm{kcal} / \mathrm{mol}\right)$, suggesting Cy7-PPG should be a stable molecule in the ground state. Overall, we concluded that widely employed PLs (e.g. carboxylates) $)^{[10,13,21-25,41]}$ should be heterolyzed from Cy7-PPG upon irradiation, especially if the effective heterolysis barriers were even lower due to higher vibrational states being accessed upon excitation. 


\section{Results and Discussion}

We thus set out to develop a synthesis towards Cy7-PPG, with $\mathbf{P L}=\mathrm{OAc}$, the archetypal PL used throughout previous studies, ${ }^{[10,13,21-24,41,42]}$ and also used in our computational evaluation (Figure 2c). Heptamethine dyes (e.g. IR-786) are classically formed via double condensation of tetramethylindolinium salts with conjugated dialdehyde reactant partners. ${ }^{[43]}$ In these cases, the synthesis of IR-786 derivatives with a meso-chloro substituent on the are facile. ${ }^{[44]}$ From here on, functionalizing the meso-carbon with various nucleophiles has been demonstrated. ${ }^{[31,44-47]}$ Furthermore, the Suzuki cross-coupling of IR-786 derivatives has also been accomplished, ${ }^{[48-50]}$ despite the low reactivity of the $\mathrm{C}-\mathrm{Cl}$ bond (Scheme 1).

Scheme 1. Attempted synthetic approaches to Cy7-PPG

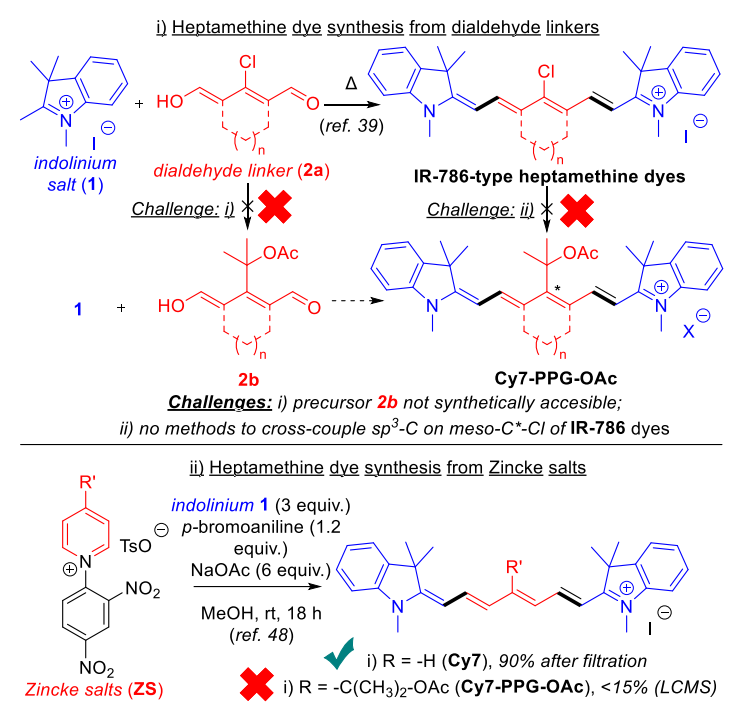

However, the synthesis of heptamethine dyes with bulky $s p^{3}$-carbon meso-substituents, such as our target molecule Cy7-PPG, remains challenging (Scheme 1). Unfortunately, our efforts to synthesize a suitable dialdehyde variant $\mathbf{2} \mathbf{b}$ with a fully substituted geminal dimethyl $s p^{3}$-carbon attached to the mesocarbon were unsuccessful. Our attempts to effect cross-coupling of IR-786 variants towards Cy7-PPG were also unsuccessful, prompting us to explore other synthetic approaches towards Cy7-PPG.

As such we turned to an alternative synthetic strategy involving activated Zincke pyridinium salts as synthetic precursors. ${ }^{[51]}$ Zincke salts have been successfully deployed as masked conjugated dialdehydes, able to react in the presence of aniline promoters, with carbon nucleophiles such as indolinium 1 to ultimately form heptamethine systems. ${ }^{[52]}$ Using this method, we successfully synthesized the parent dye Cy7 from Zincke salt ZS in excellent yield (Scheme 1). However, the method was found to perform poorly towards the desired target Cy7-PPG-OAc. The low yield shown in Scheme 1 was obtained regardless of whether the reaction was stopped at low or high conversion and was determined to be due to decomposition of the product Cy7-PPG by the combination of reactants, promoters, and solvent in this reaction mixture. The poor performance of this reaction is supported by the lack of synthetic examples of Cy7 variants with bulkier or electron-donating groups on the mesoposition from the published scope of this method, ${ }^{[52]}$ but the reason for this incompatibility is not clear. Furthermore, this reaction yielded Cy7-PPG-OAc as the iodide salt, and not the desired chloride salt. Thus, we optimized the reaction to develop a reliable, high yielding and facile synthesis of Cy7-PPGOAc with the desired chloride counterion, (Scheme 2).

The required ZS-OAC chloride salt can be conveniently accessed in two steps, with no extensive purification needed. First, commercially available 2-(4-pyridyl)-2-propanol underwent $S_{N} A r$ reaction with commercially available 2,4-dinitrobenzene, to furnish gram-scale amounts of $\mathbf{Z S}-\mathbf{O H}$ in $74 \%$ yield as $\mathrm{x}$ ray quality crystals after a single recrystallization. At this stage it was hypothesized that ZS-OH could be loaded with either an acid chloride or a carboxylic acid to serve as the PL for the photochemical evaluation. Indeed, even in the absence of a base, the bulky alcohol ZS-OH underwent smooth 
esterification in dry MeCN with acetyl chloride to yield ZS-OAc chloride salt in 95\% yield, also as x-ray quality crystals after a single-pass recrystallization. Likewise, a second method involving a Steglich esterification with $\mathrm{AcOH}$ promoted by EDC also furnished the same salt ZS-OAc, ${ }^{[53]}$ in more moderate yield.

Following the development of the ZS-OAc synthesis, we improved upon the ring-opening reaction of ZS-OAC (Scheme 1) by switching the solvent to non-protic DMF, by excluding all reaction promoters and by exchanging the indolinium salt $\mathbf{1}$ for its Fischer free base $\mathbf{1 b}$. These conditions furnish the desired Cy7-PPG-OAc as the chloride salt in $69 \%$ yield.

Scheme 2. An efficient synthesis toward Cy7-PPG, with two methods for loading ZS-OH with a payload

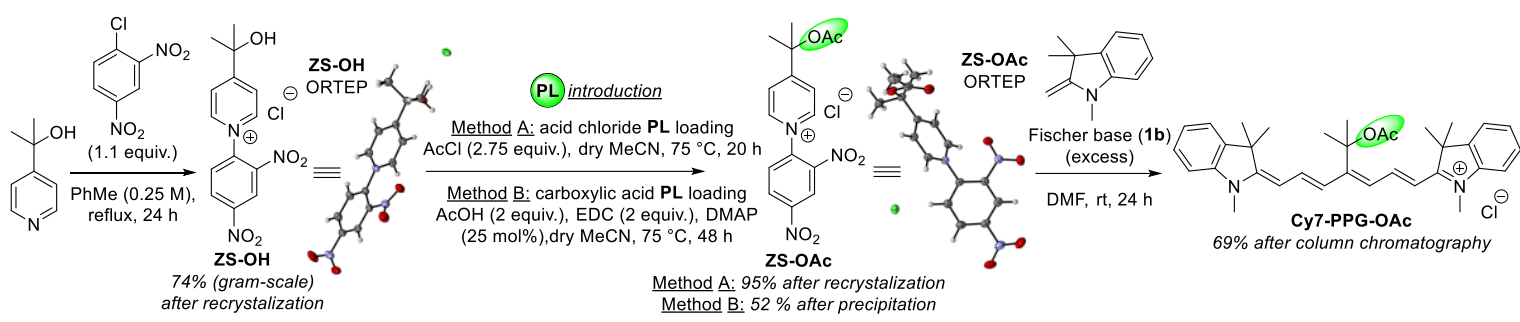

Spectroscopic evaluation of Cy7-PPG-OAc revealed its ideal properties as a photocaged compound (Figure 3): we pleasingly found Cy7-PPG-OAc to exhibit absorb at a $\lambda_{\max }$ of $746 \mathrm{~nm}$, with a high $\varepsilon$ of $2.76 \times 10^{5} \mathrm{M}^{-1} \mathrm{~cm}^{-1}$ in aqueous solvent and with good solubility at pharmacologically relevant micromolar concentrations. We also found that Cy7-PPG-OAc is stable in solution at room temperature for days in the dark and under ambient light.

Figure 3. Cy7-PPG-OAc ideal photocage properties and payload uncaging with $760 \mathrm{~nm}$ light

a) Cy7-PPG-OAc ideal photocage properties

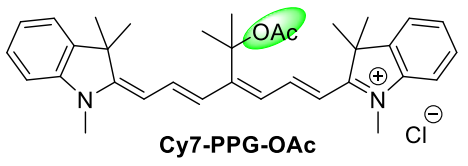

$\lambda_{\max }=746 \mathrm{~nm}, \varepsilon=276,000 \mathrm{M}^{-1} \mathrm{~cm}^{-1}$,

$\Phi_{\text {fluor }}<0.1 \%\left(\lambda_{\text {em }}=836 \mathrm{~nm}\right), \Phi_{\text {P.s. }}=0.013 \%$

(vs $\Phi_{\text {fluor }}=6.4 \%, \lambda_{\text {em }}=771 \mathrm{~nm} ; \Phi_{\text {P.S. }}=3.9 \%$ for Cy 7 )

stable in the dark and in ambient light $\left(t_{1 / 2}>\right.$ days $)$ b) Cy7-PPG-OAc ideal uncaging efficiency Ideal biological

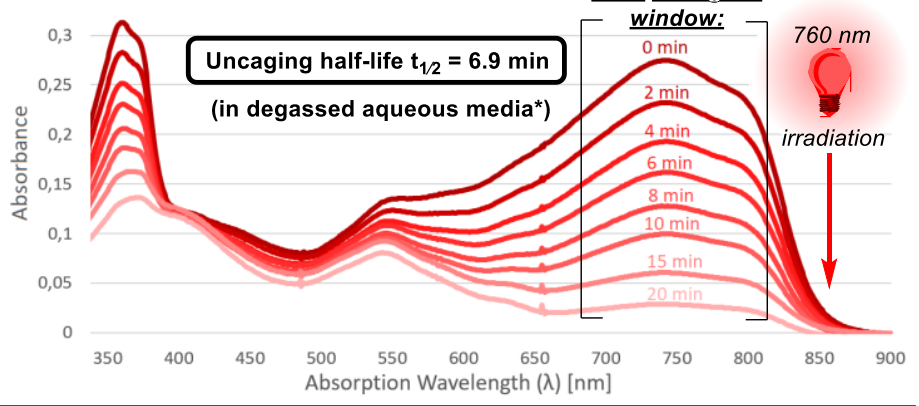

c) Cy7-PPG-OAc ideal uncaging quantum yield and photoproducts

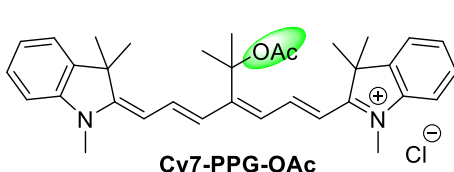

Cy7-PPG-OAc

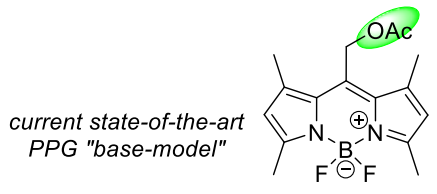

BODIPY-PPG-OAC

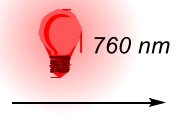

in NMR tube
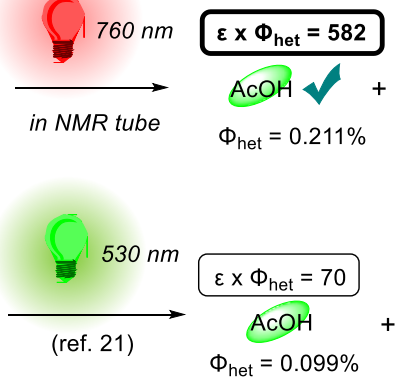

$\Phi_{\text {het }}=0.211 \%$ $\mathrm{m} / \mathrm{z}: 174.13, \lambda_{\max }<365 \mathrm{~nm}$

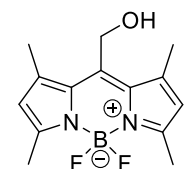

photoproduct competes for absorption with BODIPY-PPG-OAc

[a] Ideal wavelength $\lambda_{\max }$, molecular absorptivity $\varepsilon$, low fluorescence and singlet oxygen photosensitization quantum yields $\Phi_{\text {fluor }}$ and $\Phi_{\text {P.S. }}$, and high stability of Cy7-PPG-OAc; [b] UV/vis monitored uncaging of Cy7-PPG-OAc upon irradiation with $760 \mathrm{~nm}$ light ( ${ }^{*} 1 \mu \mathrm{M}$ in degassed 99:1 milli-Q $\left.\mathrm{H}_{2} \mathrm{O} / \mathrm{DMSO}\right)$; [c] NMR monitored uncaging of Cy7PPG-OAc and comparison of its uncaging cross-section $\varepsilon \times \Phi_{\text {het }}$ to current state-of-the-art BODIPY-PPG (see SI). 
We then explored other key photochemical properties of Cy7-PPG-OAc. The parent dye Cy7 is moderately fluorescent $\left(\Phi_{\text {fluor }}=6.4 \%, \lambda_{\mathrm{em}}=771 \mathrm{~nm}\right),{ }^{[34]}$ yet in sharp contrast, Cy7-PPG was found to be very weakly fluorescent $\left(\Phi_{\text {fluor }}<0.1 \%, \lambda_{\text {em }}=836 \mathrm{~nm}\right.$ ). Dye Cy7 is also a moderate oxygen photosensitizer $\left(\Phi_{\text {P.S. }}=3.9 \%\right)$ by virtue of its ISC to its $T_{1}$ state. ${ }^{[34,54]}$ However, in a direct comparison to its parent dye Cy7, Cy7-PPG-OAc was found to generate far less singlet oxygen upon excitation, with a $\Phi_{P . S .}$ of only $0.013 \%$. Indeed, similar phenomena have been observed for related dyes, where engineering a more sterically crowded heptamethine chain results in a lower the $\Phi_{\text {P.s. }}$ from the excited state of the dye, perhaps by shielding the chromophore from triplet-triplet annihilation with ambient molecular oxygen. ${ }^{[55]}$ Alternatively, the bulky geminal dimethyl moiety in Cy7-PPG may be enabling faster, non-radiative return to the ground state via the "loose bolt" effect.[56]

Encouraged by all these gratifying observations, we proceeded to investigate the uncaging of Cy7-PPG. To our delight, and fully vindicating our extensive design efforts, we observed that upon irradiation with $760 \mathrm{~nm}$ light, Cy7-PPG-OAc rapidly uncaged our model PL AcOH in a matter of minutes in degassed aqueous media (Figure $3 b$ ). We determined the heterolysis cross-section $\varepsilon \times \Phi_{\text {het }}$ for the uncaging of this moderate leaving group to be $582 \mathrm{M}^{-1} \mathrm{~cm}^{-1}$, an order of magnitude higher than the $\varepsilon \times \Phi_{\text {het }}$ of the same PL from BODIPY-PPG-OAc (see SI).[21] Interestingly, aside from successfully uncaged PL, an unexpected mixture of photoproducts derived from the PPG was observed during the photoheterolysis shown in Figure 3c. LCMS showed that after uncaging of $\mathrm{AcOH}$, the chromophore fragmented into two unsymmetrical parts which had no absorption in the NIR wavelength range. Ergo, the photolysis of Cy7PPG-OAc does not generate photoproducts that compete for photons required for the PPG's activation, as is the case for other PPGs. ${ }^{[10-12,22-24]}$

Figure 4. Cy7-PPG-OAc uncaging with $760 \mathrm{~nm}$ light inside of tissue phantoms

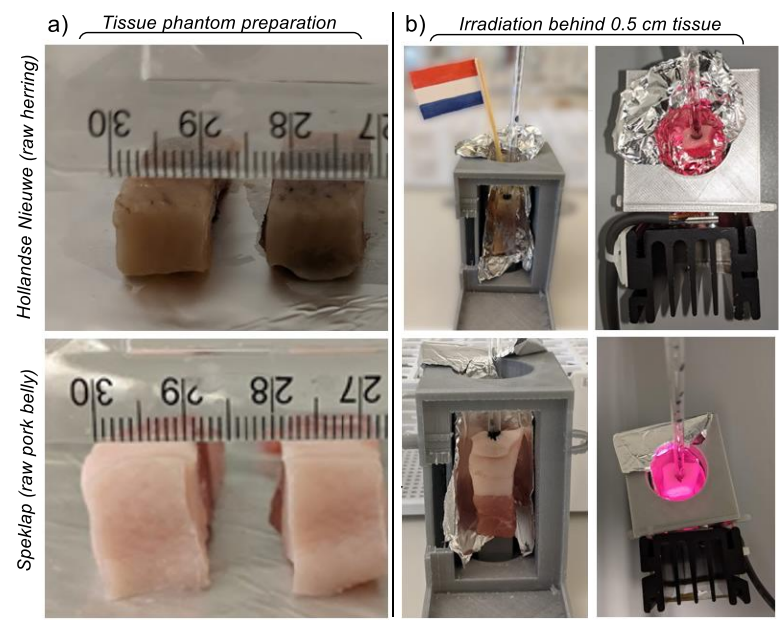

c) rate of uncaging through tissues

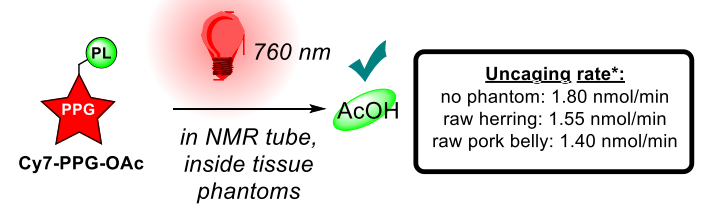

a) Preparation of $1 \mathrm{~cm}$ cuboid tissue phantoms of Hollandse Nieuwe (raw herring) and Speklap (raw pork belly); b) Mounted tissue phantoms containing a sample of Cy7-PPG-OAc (0.5 ml, $2 \mathrm{mM}$ in 1:1 $\left.\mathrm{D}_{2} \mathrm{O} / \mathrm{d}_{6}-\mathrm{DMSO}\right)$; c) Rate of PL uncaging upon $760 \mathrm{~nm}$ irradiation through the tissue phantoms.

Notably, the NIR light-induced PL release for our designed Cy7-PPG-OAc does not require oxygen, as evidence by: i) our quantum yield of uncaging $\Phi_{\text {het }}=0.211 \%$ (Figure $3 c$ ) which is an order of magnitude higher than the oxygen photosensitization quantum yield $\Phi_{\text {P.S. }}=0.013 \%$ (Figure 3a); and ii) the fact that PL uncaging occurs smoothly in degassed aqueous media (Figure $3 b$ ). This observation is further supported by our predicted computational hypothesis about the PL heterolytic step (Figure 2c). This is especially true when considering the large observed stokes shift (90 nm, Figure 3a), showing that higher 
S1 (and potentially T1) vibrational states are accessed upon excitation, lowering the effective barriers for PL heterolysis upon excitation.

Finally, with Cy7-PPG in hand, we sought to demonstrate our ability to uncage the Cy7-PPG-OAc within complex tissue phantoms (Figure 4). To this end, samples of Cy7-PPG-OAc were irradiated with 760 nm light inside a series of a readily available Dutch food samples, namely Hollandse Nieuwe (raw Dutch Herring) and Speklap (raw pork belly). Unsurprisingly, $760 \mathrm{~nm}$ light passes unfettered through roughly $0.5 \mathrm{~cm}$ of these tissues to induce heterolysis of $\mathrm{AcOH}$ from Cy7-PPG-OAc, with little impact on photolytic rate: $86 \%$ and $78 \%$ uncaging rate for the fish and porcine tissue, respectively, versus $760 \mathrm{~nm}$ irradiation without a tissue phantom.

\section{Conclusion}

In summary, we reported here the computational design of a new NIR-triggered PPG with ideal properties for use in biomedical applications. We demonstrate that Cy7-PPG is efficiently synthesized from commercial precursors, and we show that PLs can be readily loaded using two different synthetic methods.

Cy7-PPG is the most red-shifted $\left(\lambda_{\max }=746 \mathrm{~nm}\right)$ and most strongly absorbing $\left(\varepsilon=276,000 \mathrm{M}^{-1} \mathrm{~cm}^{-1}\right)$ heterolytic PPG reported to date. ${ }^{[12,21,23,41]}$ The overall efficiency $\left(\varepsilon \times \Phi_{\text {het }}=582 \mathrm{M}^{-1} \mathrm{~cm}^{-1}\right.$ at $\left.746 \mathrm{~nm}\right)$ of Cy7-PPG-OAc is within the ideal range $\left(>100 \mathrm{M}^{-1} \mathrm{~cm}^{-1}\right),{ }^{[12]}$ and is an order of magnitude higher than that of the "base-model" of "state-of-the-art" BODIPY-PPGs. ${ }^{[12,13,21,23,41]}$ Cy7-PG is also soluble in aqueous media at pharmacological concentrations. Furthermore our redesign of Cy7 into Cy7-PPG has ablated two photochemical pathways that are seen as less-than-desirable in PPGs: i) fluorescence, which competes with payload heterolysis; and ii) oxygen sensitization, a process that results in PPG phototoxicity. ${ }^{12]}$ Cy7-PPG is thus a NIR-photocage system whose release mechanism is entirely uncoupled from singlet oxygen phototoxicity. Finally, we have demonstrated that Cy7-PPG can be activated within model complex animal tissues using NIR light.

This advancement in NIR-PPG technology paves the way towards clinical application of light for local activation of therapeutic effects in deep tissue, with unprecedented spatial and temporal precision.

\section{Acknowledgements}

We are grateful for the generous funding support to G.A. (EMBO LTF-232-2020 Postdoctoral Fellowship), to B.L.F. (ERC Advanced Investigator Grant No. 694345; and the Ministry of Education, Culture and Science of The Netherlands Gravitation Program No. 024.001.035), and to W.S. (The Netherlands Organization for Scientific Research/NWO, VIDI grant no. 723.014.001).

We are grateful to J.L. Sneep for collecting high-resolution mass spectrometry data for all newly reported compounds.

\section{Author Contributions}

The manuscript was written through contributions of all authors. G.A. conceived the study, executed computations, performed synthesis, and characterized all compounds and their photochemistry. A.M.S. conceived synthetic steps and was involved in photochemical characterization. A.M. performed x-ray crystallographic analysis. W.S. and B.L.F assisted in conceiving the study. All authors were involved in the manuscript writing process and have given approval to the final version of the manuscript.

\section{Corresponding Authors}

* Dr. Georgios Alachouzos - Centre for Systems Chemistry, Stratingh Institute for Chemistry, Faculty for Science and Engineering, University of Groningen, Nijenborgh 4, 9747 AG Groningen, The Netherlands; https://orcid.org/0000-0002-3058-2246; Email: g.alachouzos@rug.nl

* Prof. Wiktor Szymanski - Department of Radiology, Medical Imaging Center, University Medical Center Groningen, University of Groningen, Hanzeplein 1, 9713 GZ Groningen, The Netherlands; https://orcid.org/0000-0002-9754-9248; E-mail:w.szymanski@umcg.nl 
* Prof. Ben L. Feringa - Centre for Systems Chemistry, Stratingh Institute for Chemistry, Faculty for Science and Engineering, University of Groningen, Nijenborgh 4, 9747 AG Groningen, The Netherlands; ; https://orcid.org/0000-0003-0588-8435; Email: b.l.feringa@rug.nl

\section{References}

[1] M. M. Lerch, M. J. Hansen, G. M. van Dam, W. Szymanski, B. L. Feringa, Angew. Chem. Int. Ed. 2016, 55, 10978-10999.

[2] K. Hüll, J. Morstein, D. Trauner, Chem. Rev. 2018, 118, 10710-10747.

[3] S. Jia, E. M. Sletten, ACS Chem. Biol. 2021, acschembio.1c00518.

[4] W. A. Velema, W. Szymanski, B. L. Feringa, J. Am. Chem. Soc. 2014, 136, 2178-2191.

[5] M. J. Fuchter, J. Med. Chem. 2020, 63, 11436-11447.

[6] M. Ricart-Ortega, J. Font, A. Llebaria, Mol. Cell. Endocrinol. 2019, 488, 36-51.

[7] K. Long, H. Han, W. Kang, W. Lv, L. Wang, Y. Wang, L. Ge, W. Wang, J. Nanobiotechnology 2021, $19,357$.

[8] C. Brieke, F. Rohrbach, A. Gottschalk, G. Mayer, A. Heckel, Angew. Chemie Int. Ed. 2012, 51, 8446-8476.

[9] M. W. H. Hoorens, W. Szymanski, Trends Biochem. Sci. 2018, 43, 567-575.

[10] T. Šolomek, J. Wirz, P. Klán, Acc. Chem. Res. 2015, 48, 3064-3072.

[11] M. J. Hansen, W. A. Velema, M. M. Lerch, W. Szymanski, B. L. Feringa, Chem. Soc. Rev. 2015, 44, 3358-3377.

[12] P. Štacko, T. Šolomek, Chimia (Aarau). 2021, 75, 873-881.

[13] R. Weinstain, T. Slanina, D. Kand, P. Klán, Chem. Rev. 2020, 120, 13135-13272.

[14] I. M. Welleman, M. W. H. Hoorens, B. L. Feringa, H. H. Boersma, W. Szymański, Chem. Sci. 2020, 11, 11672-11691.

[15] A. E. Mangubat-Medina, Z. T. Ball, Chem. Soc. Rev. 2021, 50, 10403-10421.

[16] K. Long, Y. Yang, W. Lv, K. Jiang, Y. Li, A. C. Y. Lo, W. C. Lam, C. Zhan, W. Wang, Adv. Sci. 2021, 8, 2101754.

[17] Y. Li, W. Lv, L. Wang, Y. Zhang, L. Yang, T. Wang, L. Zhu, Y. Wang, W. Wang, Nano Res. 2021, 14, 2630-2636.

[18] A. M. Smith, M. C. Mancini, S. Nie, Nat. Nanotechnol. 2009, 4, 710-711.

[19] L. N. Lameijer, D. Ernst, S. L. Hopkins, M. S. Meijer, S. H. C. Askes, S. E. Le Dévédec, S. Bonnet, Angew. Chemie Int. Ed. 2017, 56, 11549-11553.

[20] V. H. S. van Rixel, V. Ramu, A. B. Auyeung, N. Beztsinna, D. Y. Leger, L. N. Lameijer, S. T. Hilt, S. E. Le Dévédec, T. Yildiz, T. Betancourt, M. B. Gildner, T. W. Hudnall, V. Sol, B. Liagre, A. Kornienko, S. Bonnet, J. Am. Chem. Soc. 2019, 141, 18444-18454.

[21] P. Shrestha, K. C. Dissanayake, E. J. Gehrmann, C. S. Wijesooriya, A. Mukhopadhyay, E. A. Smith, A. H. Winter, J. Am. Chem. Soc. 2020, 142, 15505-15512.

[22] P. Klán, T. Šolomek, C. G. Bochet, A. Blanc, R. Givens, M. Rubina, V. Popik, A. Kostikov, J. Wirz, Chem. Rev. 2013, 113, 119-191.

[23] T. Slanina, P. Shrestha, E. Palao, D. Kand, J. A. Peterson, A. S. Dutton, N. Rubinstein, R. Weinstain, A. H. Winter, P. Klán, J. Am. Chem. Soc. 2017, 139, 15168-15175.

[24] K. Sitkowska, B. L. Feringa, W. Szymański, J. Org. Chem. 2018, 83, 1819-1827.

[25] K. Sitkowska, M. F. Hoes, M. M. Lerch, L. N. Lameijer, P. van der Meer, W. Szymański, B. L. 
Feringa, Chem. Commun. 2020, 56, 5480-5483.

[26] Q. Lin, L. Yang, Z. Wang, Y. Hua, D. Zhang, B. Bao, C. Bao, X. Gong, L. Zhu, Angew. Chem. Int. Ed. 2018, 57, 3722-3726.

[27] H. S. Yu, X. He, S. L. Li, D. G. Truhlar, Chem. Sci. 2016, 7, 5032-5051.

[28] J. Zheng, X. Xu, D. G. Truhlar, Theor. Chem. Acc. 2011, 128, 295-305.

[29] A. V. Marenich, C. J. Cramer, D. G. Truhlar, J. Phys. Chem. B 2009, 113, 6378-6396.

[30] J. T. Alander, I. Kaartinen, A. Laakso, T. Pätilä, T. Spillmann, V. V. Tuchin, M. Venermo, P. Välisuo, Int. J. Biomed. Imaging 2012, 2012, 1-26.

[31] L. Feng, W. Chen, X. Ma, S. H. Liu, J. Yin, Org. Biomol. Chem. 2020, 18, 9385-9397.

[32] I. Dika, J.-P. Malval, O. Soppera, V. Bardinal, D. Barat, C. Turck, A. Spangenberg, A. Bruyant, Chem. Phys. Lett. 2011, 515, 91-95.

[33] L. Jiao, F. Song, J. Cui, X. Peng, Chem. Commun. 2018, 54, 9198-9201.

[34] S. S. Matikonda, G. Hammersley, N. Kumari, L. Grabenhorst, V. Glembockyte, P. Tinnefeld, J. Ivanic, M. Levitus, M. J. Schnermann, J. Org. Chem. 2020, 85, 5907-5915.

[35] D. Li, B. D. Smith, Chem. - A Eur. J. 2021, 27, 14535-14542.

[36] A. P. Gorka, R. R. Nani, J. Zhu, S. Mackem, M. J. Schnermann, J. Am. Chem. Soc. 2014, 136, 14153-14159.

[37] R. R. Nani, A. P. Gorka, T. Nagaya, H. Kobayashi, M. J. Schnermann, Angew. Chemie Int. Ed. 2015, 54, 13635-13638.

[38] K. Fajans, Naturwissenschaften 1923, 11, 165-172.

[39] K. Fajans, G. Joos, Zeitschrift Phys. 1924, 23, 1-46.

[40] K. Fajans, Zeitschrift für Krist. - Cryst. Mater. 1924, 61, 18-48.

[41] J. A. Peterson, C. Wijesooriya, E. J. Gehrmann, K. M. Mahoney, P. P. Goswami, T. R. Albright, A. Syed, A. S. Dutton, E. A. Smith, A. H. Winter, J. Am. Chem. Soc. 2018, 140, 7343-7346.

[42] K. Sitkowska, M. F. Hoes, M. M. Lerch, L. N. Lameijer, P. van der Meer, W. Szymański, B. L. Feringa, Chem. Commun. 2020, 56, 5480-5483.

[43] G. A. Reynolds, K. H. Drexhage, J. Org. Chem. 1977, 42, 885-888.

[44] N. Narayanan, G. Patonay, J. Org. Chem. 1995, 60, 2391-2395.

[45] X. Peng, F. Song, E. Lu, Y. Wang, W. Zhou, J. Fan, Y. Gao, J. Am. Chem. Soc. 2005, 127, 4170-4171.

[46] F. Song, X. Peng, E. Lu, R. Zhang, X. Chen, B. Song, J. Photochem. Photobiol. A Chem. 2004, 168, 53-57.

[47] J. H. Flanagan, S. H. Khan, S. Menchen, S. A. Soper, R. P. Hammer, Bioconjug. Chem. 1997, $8,751-756$.

[48] D. Su, C. L. Teoh, A. Samanta, N.-Y. Kang, S.-J. Park, Y.-T. Chang, Chem. Commun. 2015, $51,3989-3992$.

[49] H. Lee, J. C. Mason, S. Achilefu, J. Org. Chem. 2008, 73, 723-725.

[50] Y. Liu, D. Qiu, H. Pan, M. Li, H. Chen, H. Li, J. Photochem. Photobiol. A Chem. 2018, 364, 151-158.

[51] T. Zincke, G. Heuser, W. Möller, Justus Liebig's Ann. der Chemie 1904, 333, 296-345.

[52] L. Štacková, P. Štacko, P. Klán, J. Am. Chem. Soc. 2019, 141, 7155-7162. 
[53] B. Neises, W. Steglich, Angew. Chem. Int. Ed. 1978, 17, 522-524.

[54] H.-J. Adick, R. Schmidt, H.-D. Brauer, J. Photochem. Photobiol. A Chem. 1990, 54, 27-30.

[55] D. Li, C. L. Schreiber, B. D. Smith, Angew. Chemie Int. Ed. 2020, 59, 12154-12161.

[56] Nicholas Turro, Modern Molecular Photochemistry of Organic Molecules, 2010. 